\title{
Benefit of wearable blood pressure monitoring device in Society 5.0
}

\author{
Takuya Kishi $^{1}$
}

Received: 18 November 2021 / Accepted: 24 November 2021 / Published online: 13 January 2022

(c) The Author(s), under exclusive licence to The Japanese Society of Hypertension 2022

Nobody doubt that hypertension is the primary therapeutic target to prevent cardiovascular events.

Especially, frequent daytime blood pressure (BP) measurements during daily activities over several days may be critical for detecting daytime BP risk. Self-measured home BP monitoring (HBPM) and ambulatory BP monitoring $(\mathrm{ABPM})$ are recommended among $\mathrm{BP}$ measurements. However, HBPM can underestimate the risk of masked hypertension, and ABPM cannot be performed frequently. We do not have an adequate BP monitoring device that could measure daytime BP during activities of daily life. In these aspects, we have expected less restrictive wearable BP monitoring devices I the era of digital management of hypertension. Among the wearable BP monitoring devices, Kario et al previously validated the HeartGuide ${ }^{\circledR}$, a wristwatch-type wearable BP monitoring device, and verified its accuracy under the ambulatory condition in a comparison study of subjects simultaneously equipped with ABPM [1-3]. It is necessary to assess the benefits of wearable BP monitoring devices to prevent hypertensive organ damages.

In this issue, Kario et al. [4] evaluated the relationship between BP taken by a new wrist-cuff oscillometric wearable BP monitoring device and left ventricular mass index measured by cardiac magnetic resonance imaging (cMRILVMI) in 50 hypertensive patients (mean age 60.5 \pm 8.9 years, $92.0 \%$ men, $96 \%$ treated for hypertension) with regular employment. In 2105 patients with wearable BP measurements (home BP: 747 [morning: 409, evening: 338], ambulatory condition: 1358 [worksite: 942]) were collected. The average of all wearable systolic BP (129.8 \pm $11.0 \mathrm{mmHg}$ ) was weakly correlated with cMRI-LVMI. Morning home wearable systolic BP average $(128.5 \pm 13.8$ $\mathrm{mmHg}$ ) was significantly correlated with cMRI-LVMI,

Takuya Kishi

tkishi@iuhw.ac.jp

1 Graduate School of Medicine (Cardiology), International University of Health and Welfare, Okawa, Japan but ambulatory wearable systolic BP average (132.5 \pm $12.7 \mathrm{mmHg}$ ) was not. The averages of the highest three values of all wearable systolic BP $(153.3 \pm 13.9 \mathrm{mmHg})$ and ambulatory wearable systolic BP $(152.9 \pm 13.9 \mathrm{mmHg})$ were $16 \mathrm{mmHg}$ higher than that of the morning home wearable systolic BP $(137.0 \pm 15.9 \mathrm{mmHg})$. Those peak values were significantly correlated with cMRI-LVMI. Considering these results, Kario et al concluded that an increased number of wearable BP measurements might add to the clinical value of these measurements as a complement to the guideline-recommended home BP measurements.

This is the first study examining the association between wearable wrist-cuff oscillometric BP monitoring devices and hypertensive organ damages in patients with hypertension. The most important new finding was that the wearable systolic BP at home according to the guideline-recommended standardized measurements protocol was more strongly correlated with cMRI-LVMI than that at home and under an ambulatory condition. In this study, the averages of the ambulatory wearable systolic BP and worksite wearable systolic BP values were higher than the average of the guideline-recommended home wearable systolic BP by 7.9 and $8.8 \mathrm{mmHg}$, respectively. Moreover, the worksite wearable systolic BP was higher than the morning home wearable systolic BP by $4.9 \mathrm{mmHg}$. However, the correlation of ambulatory wearable SBP and morning BP measured by ABPM with cMRI-LVMI was not significant, while that of home (morning average and morningevening average) wearable SBP with cMRI-LVMI was significant. These findings provide us the meaningful clinical implications that wearable BP might be a more sensitive measure for evaluating organ damage and that the repeated morning BP measurement according to a standardized protocol is essential for hypertension management.

The secondary important new finding was that the peak wearable systolic BP during ambulatory measurements was significantly higher than those during home morning measurements, and that both the wearable peak systolic BP index measured under a home condition and that measured under an ambulatory condition were significantly correlated with cMRI-LVMI. Kario et al discussed that automatically 
measured ABPM captured BP under a wider range of conditions compared with ambulatory wearable BP, which can lead to greater BP variability and poor correlation. These results clearly showed that wearable BP may be more sensitive than ABPM-measured BP for assessing cardiovascular risk during daily activity.

Although there are several limitations and further studies are necessary, the newly developed wrist-cuff oscillometric wearable BP monitoring device, HeartGuide, was feasible and acceptable for working adults, and was able to detect the BP elevation during daily activity. These benefits contribute to the prevention of hypertensive organ damages. In Society5.0, wearable BP monitoring devices will strengthen the established therapeutics for hypertension [5].

\section{Compliance with ethical standards}

Conflict of interest The author declares no competing interests.

Publisher's note Springer Nature remains neutral with regard to jurisdictional claims in published maps and institutional affiliations.

\section{References}

1. Kario K, Shimbo D, Tomitani N, Kanegae H, Schwartz JE, Williams B. The first study comparing a wearable watch-type blood pressure monitor with a conventional ambulatory blood pressure monitor on in-office and out-of-office settings. J Clin Hypertens. 2020;22:135-41.

2. Kuwabara M, Harada K, Hishiki Y, Kario K. Validation of a wristtype home nocturnal blood pressure monitor in the sitting and supine position according to the ANSI/AAMI/ISO81060-2:2013 guidelines: Omron HEM-9600T. J Clin Hypertens. 2019;21:463-9.

3. Kuwabara M, Harada K, Hishiki Y, Ohkubo T, Kario K, Imai Y. Validation of a wrist-type home nocturnal blood pressure monitor in the sitting and supine position according to the ANSI/AAMI/ ISO81060-2:2013 guidelines: Omron HEM-9601T. J Clin Hypertens. 2020;22:970-8.

4. Kario K, Tomitani N, Morimoto T, Kanegae H, Lacy P, and Williams B. Relationshio between blood pressure repeatedly measured by a wrist-cuff oscillometric wearable blood pressure monitoring device and left ventricular mass index in working hypertension patients. Hypertens Res. https://doi.org/10.1038/s41440-02100758-3.

5. Kario K. Management of hypertension in the digital era: small wearable monitoring devices for remote blood pressure monitoring. Hypertension. 2020;76:640-50. 